\title{
Difficulties in the description of Drell-Yan processes at low invariant mass and high transverse momentum
}

\author{
Alessandro Bacchetta, ${ }^{a b}$ Giuseppe Bozzi, ${ }^{a b}$ Martin Lambertsen, ${ }^{c}$ Fulvio Piacenza ${ }^{* a b}$ \\ Julius Steiglechner, ${ }^{c}$ Werner Vogelsang ${ }^{c}$ \\ ${ }^{a}$ Dipartimento di Fisica, Università di Pavia, via Bassi 6, I-27100 Pavia \\ ${ }^{b}$ INFN Sezione di Pavia, via Bassi 6, I-27100 Pavia \\ ${ }^{c}$ Institute for Theoretical Physics, Tübingen University, Auf der Morgenstelle 14, D-72076 \\ Tübingen, Germany \\ E-mail: alessandro.bacchetta@unipv.it, giuseppe.bozzi@unipv.it, \\ lambertsen@tphys.physik.uni-tuebingen.de, \\ fulvio.piacenza01@universitadipavia.it, steiglju@gmail.com, \\ werner.vogelsang@uni-tuebingen.de
}

\begin{abstract}
We study the Drell-Yan cross section differential with respect to the transverse momentum of the produced lepton pair. We consider data with moderate invariant mass $Q$ of the lepton pair, between $4.5 \mathrm{GeV}$ and $13.5 \mathrm{GeV}$, and similar (although slightly smaller) values of the transverse momentum $q_{T}$. We approach the problem by deriving predictions based on standard collinear factorization, which are expected to be valid toward the high- $q_{T}$ end of the spectrum and to which any description of the spectrum at lower $q_{T}$ based on transverse-momentum dependent parton distributions ultimately needs to be matched. We find that the collinear framework predicts cross sections that in most cases are significantly below available data at high $q_{T}$. We discuss additional perturbative and possible non-perturbative effects that increase the predicted cross section, but not by a sufficient amount.
\end{abstract}

23rd International Spin Physics Symposium - SPIN2018 -

10-14 September, 2018

Ferrara, Italy

\footnotetext{
* Speaker.
} 


\section{Introduction}

The transverse-momentum dependent Drell-Yan (DY) cross section has been considered in different studies [1, 2, 3, 4, 5, 6, 7, 8] for the determination of Transverse Momentum Distributions (TMDs). However, TMDs can only describe the low transverse momentum region, $q_{T} \ll Q$, and, concerning fixed-target experiments, there is no attempt to address the region $\Lambda_{\mathrm{QCD}} \ll q_{T} \lesssim Q$, where theoretically one expects a transition from TMDs to fixed-order collinear factorization. In this talk, we show that fixed-order predictions significantly underestimate the available fixed-target data even at the highest accessible values of $q_{T}$. To understand this discrepancy, we investigate two possible extensions of the collinear framework: the resummation of high- $q_{T}$ threshold logarithms, and intrinsic transverse-momentum smearing. Even if they are relevant for some kinematics, these effects do not seem to give a general solution to the problem.

It is interesting that also for semi-inclusive deep inelastic scattering (SIDIS) processes ([9]) large discrepancies were found between fixed-order calculations and experimental data at high $q_{T}$. Understanding the origin of these issues, besides being relevant by itself, is necessary in order to have full control of TMD physics and its matching to collinear physics.

The results we present here are available in a more extensive form in Ref. [10].

\section{Collinear factorization and comparison to low energy data}

Although there is a generally good agreement between fixed-order predictions and collider Drell-Yan data (see, for instance, Fig. 10 and Fig. 11 in [11] for a comparison to ATLAS data), this is not the case for low energy experiments. In this regime, data seem to be well described when integrated over $q_{T}$ (i.e. when the cross section is differential in rapidity and invariant mass): this is the case of the Fermilab E288 [12] and E866 [13, 14] data sets, commonly used in global PDF fits (like, e.g., $[15,16])$. When comparing the $q_{T}$-differential cross section to collinear factorization predictions, the situation is as shown in Figs. 1 and 2. The predictions are known not to be reliable for $q_{T} \ll Q$, where resummation of $q_{T}$-logarithms is needed. However, data are largely underestimated even at the highest accessible values of $q_{T}\left(q_{T} \sim Q\right.$ for the case of E866), where fixed-order calculations are expected to be valid. The fact that, for $q_{T} \gtrsim 2.5 \mathrm{GeV}$, the NLO uncertainty band in Fig. 1 overlaps with (and is eventually included in) the LO uncertainty band indeed provides some indication that perturbation theory is well-behaved for this process ${ }^{1}$. The reach in $q_{T}$ of E288 data is somewhat smaller. However, in Fig. 2 we also plot the asymptotic expansion of the resummed calculation, as this should give a rough guide concerning the region where the fixed-order calculation may start to become reliable [17]: ideally, when $q_{T}$ is large enough that the difference between the fixed-order and asymptotic calculations (the so-called " $Y$ term") exceeds the full (" $W+Y$ ") cross section, one should switch from $W+Y$ to the fixed-order result to obtain more reliable predictions. This occurs for $q_{T}$ values around 1-2 GeV in the present case. The disagreement between theory and data is general for data with center-of-mass energy of the order of few tens of $\mathrm{GeV}$ (see [10] for more plots).

\footnotetext{
${ }^{1}$ On the other hand, we also observe that the NLO scale uncertainty band is only marginally more narrow than the LO one.
} 

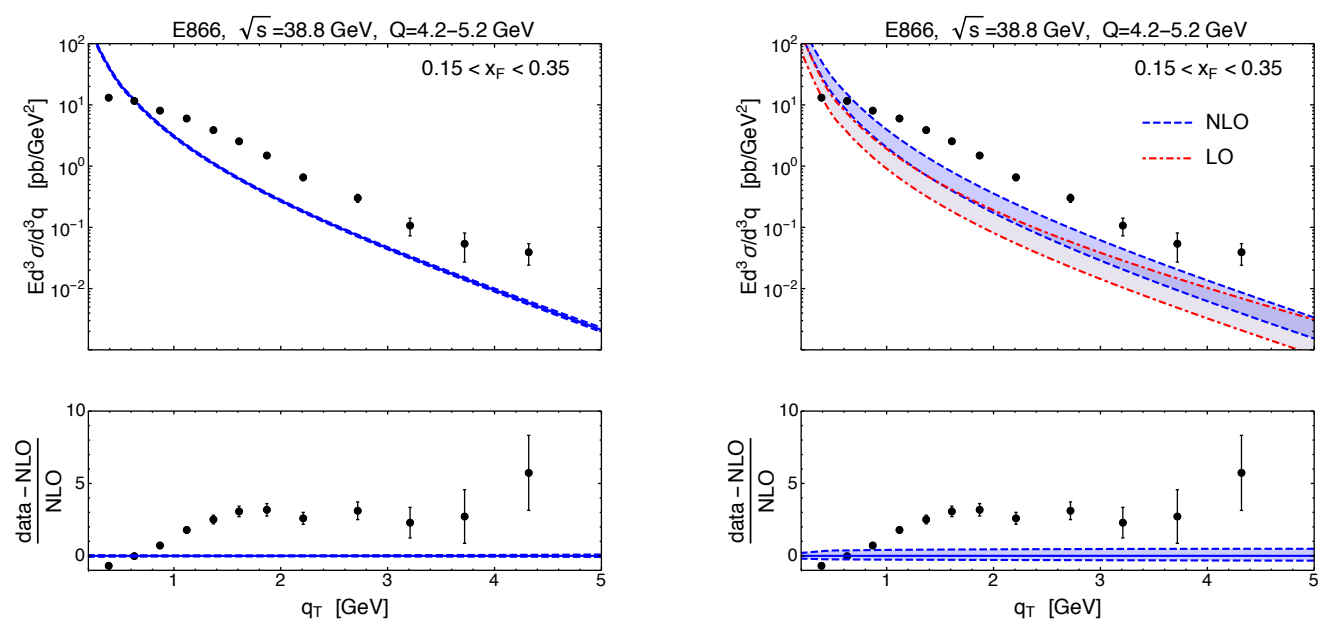

Figure 1: Transverse-momentum distribution of Drell-Yan di-muon pairs at $\sqrt{s}=38.8 \mathrm{GeV}$ in a selected invariant mass range and Feynman- $x$ range: experimental data from Fermilab E866 (hydrogen target) [14] compared to LO QCD and NLO QCD results. Left: NLO QCD $\left(\mathscr{O}\left(\alpha_{s}^{2}\right)\right)$ calculation with central values of the scales $\mu_{R}=\mu_{F}=Q=4.7 \mathrm{GeV}$, including a 90\% confidence interval from the CT14 PDF set [18]. Right: LO QCD and NLO QCD theoretical uncertainty bands obtained by varying the renormalization and factorization scales independently in the range $Q / 2<\mu_{R}, \mu_{F}<2 Q$.

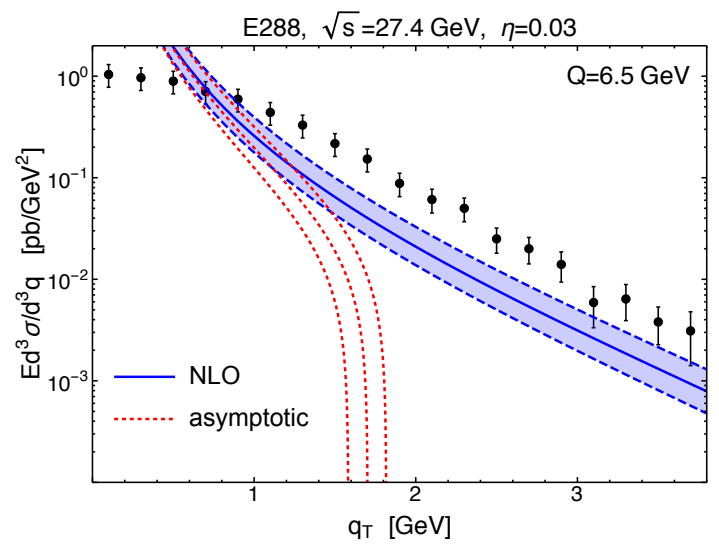

Figure 2: Experimental data from E288 [12] vs. NLO QCD predictions (full line with blue band). The NLO asymptotic expansion of the resummed result is also shown (red dotted lines). The bands are given by scale variations, as in Fig. 1, while central values correspond to $\mu_{R}=\mu_{F}=Q$. 


\section{Threshold resummation}

As we have seen in Fig. 1, the NLO corrections to the $q_{T}$-differential cross sections are quite sizable. It is therefore important to investigate in how far beyond-NLO perturbative corrections might be relevant for obtaining a better agreement with the data. For the kinematics relevant for the Fermilab experiments, the invariant mass and transverse momentum of the Drell-Yan pair are such that the production is relatively close to partonic threshold, where a new class of logarithms (separate from that mentioned above at low $q_{T}$ ) arises. The summation of these logarithms to all orders is known as threshold resummation. To be more specific, let us consider the differential cross section for $h_{1} h_{2} \rightarrow \ell^{-} \ell^{+} X$ in collinear factorization (for simplicity integrated over $y$ ):

$$
\begin{aligned}
\frac{d \sigma}{d Q^{2} d q_{T}^{2}} & =\sum_{a, b} \int_{0}^{1} d x_{1} f_{a / h_{1}}\left(x_{1}, \mu_{F}^{2}\right) \int_{0}^{1} d x_{2} f_{b / h_{2}}\left(x_{2}, \mu_{F}^{2}\right) \frac{d \hat{\sigma}_{a b}}{d Q^{2} d q_{T}^{2}} \\
& \equiv \frac{\sigma_{0}}{q_{T}^{2} Q^{2}} \sum_{a, b} \int_{y_{T}^{2}}^{1} d x_{1} f_{a / h_{1}}\left(x_{1}, \mu_{F}^{2}\right) \int_{y_{T}^{2} / x_{1}}^{1} d x_{2} f_{b / h_{2}}\left(x_{2}, \mu_{F}^{2}\right) \omega_{a b}\left(\hat{y}_{T}, r, \frac{\mu_{F}^{2}}{Q^{2}}, \frac{\mu_{R}^{2}}{Q^{2}}, \alpha_{s}\left(\mu_{R}^{2}\right)\right),
\end{aligned}
$$

where $\sigma_{0}=4 \pi \alpha^{2} /\left(9 Q^{2}\right), f_{a / h_{1}}$ and $f_{b / h_{2}}$ are the PDFs, and where $\hat{s}=s x_{1} x_{2}$ is the partonic centerof-mass energy squared. In the second line we have written out the variables that the dimensionless hard-scattering functions $\omega_{a b}$ may depend on:

$$
\hat{y}_{T} \equiv \frac{q_{T}+m_{T}}{\sqrt{\hat{s}}}, r \equiv \frac{q_{T}}{m_{T}},
$$

where $\hat{y}_{T} \leq 1$. For $\hat{y}_{T} \rightarrow 1$ the partonic center-of-mass energy is just sufficient to produce the lepton pair with mass $Q$ and transverse momentum $q_{T}$. Therefore, $\hat{y}_{T}=1$ sets a threshold for the process. As is well known [19, 20], the partonic cross sections receive large logarithmic corrections near this threshold. At the $k$ th order of perturbation theory for the $\omega_{a b}$, there are logarithmically enhanced contributions of the form $\alpha_{s}^{k} \ln ^{m}\left(1-\hat{y}_{T}^{2}\right)$, with $m \leq 2 k$. These logarithmic terms are due to soft and/or collinear gluon radiation and dominate the perturbative expansion when the process is kinematically close to the partonic threshold. We note that $\hat{y}_{T}$ becomes especially large when the partonic momentum fractions approach their lower integration limits. Since the PDFs rise steeply towards small argument, this enhances the relevance of the threshold regime, and the soft-gluon effects are relevant even when the hadronic center-of-mass energy is much larger than the produced transverse mass and transverse momentum of the final state.

We note that large corrections from threshold resummation have been found previously in purely hadronic single-inclusive processes such as $p p \rightarrow \pi X[21,22]$, which motivates a corresponding study for the high- $q_{T}$ Drell-Yan cross section $p p \rightarrow \gamma^{*} X \rightarrow \ell^{+} \ell^{-} X$ that will be carried out in this section. The relevant formalism has been developed in Refs. [23, 24, 25, 26, 27], although in most of these papers only fixed-order (NNLO) expansions of the resummed cross sections have been considered, and in [28] for the closely related high- $q_{T}$ Higgs production cross section.

We follow here the approach taken in the latter reference. For details, we refer the reader to [10]. Our numerical results are shown in Figs. 3 and 4, for a fixed value of Q and three values of $\sqrt{s}$. We have chosen $\mu_{F}=\mu_{R}=Q$. We first notice that the NLO expansion of the resummed formula (black dashed curve) accurately reproduces the NLO result (blue solid curve, with uncertainty bands). This provides some confidence that threshold resummation correctly describes the 
dominant parts of the cross section to all orders, and that subleading contributions not addressed by resummation are reasonably small. In the left part of Fig. 3 we also show the scale uncertainty band for the NLL matched result (red dot-dashed curve), which is barely broad enough to be visible. Evidently, resummation leads to a strong reduction in scale dependence, as one would expect from a result that incorporates the dominant contributions to the cross section at all orders.

Overall, we find a further significant increase of the cross section due to NLL resummation, with respect to the NLO results. The enhancement is more pronounced for the case of E288 than for E866 since, for a given $Q$, at E288 energy one is closer to threshold because of the lower c.m.s. energy. However, despite the increase, the NLL result unfortunately still remains well below the E288 and E866 experimental data at high $q_{T}$. We thus conclude that NLL high- $q_{T}$ threshold resummation is not able to lead to a satisfactory agreement with the data.
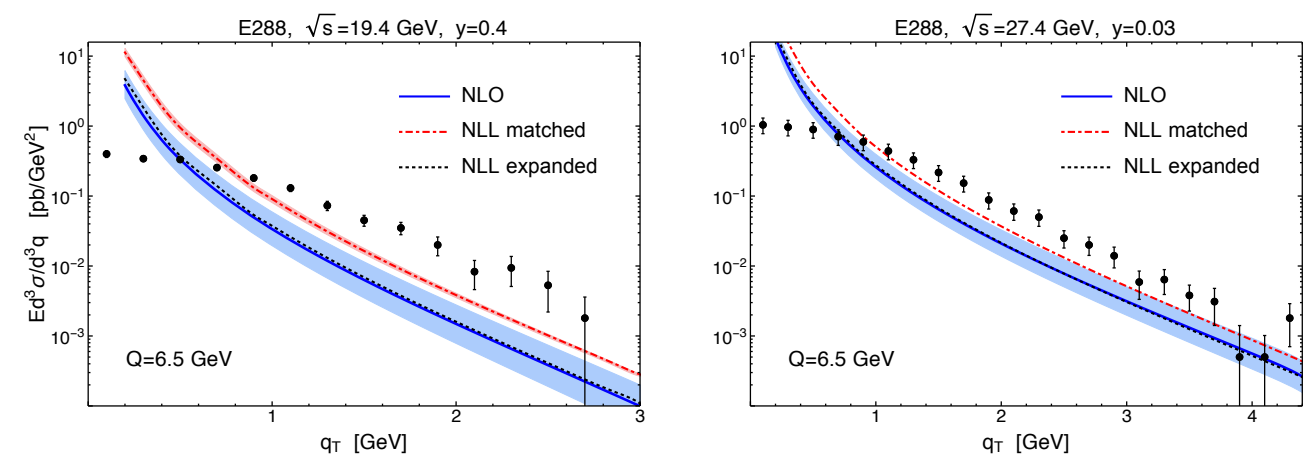

Figure 3: E288: experimental data vs. threshold-resummed predictions at NLL+NLO QCD for two different rapidity bins and two different center-of-mass energies.

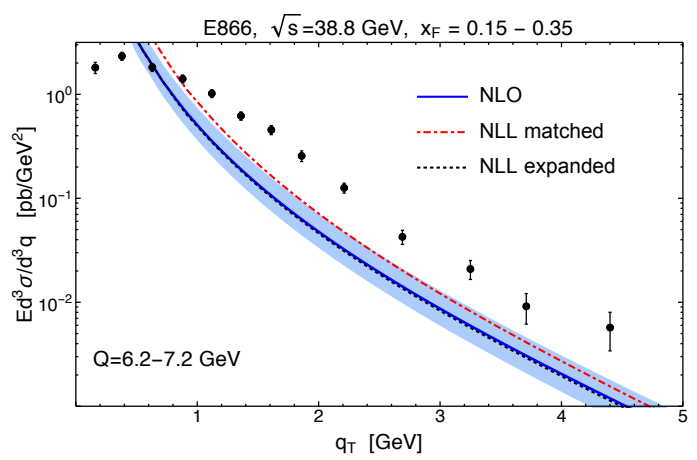

Figure 4: E866: experimental data vs. threshold-resummed predictions at NLL+NLO QCD for a selected $\left(x_{F}, Q\right)$ bin.

\section{Intrinsic- $k_{T}$ smearing and power corrections}

The factorized cross section given in Eq. (3.1) receives corrections that are suppressed by 
inverse powers of $Q \sim q_{T}$. Little is known so far about the structure and size of such power corrections for the high- $q_{T}$ Drell-Yan cross section. It is an interesting question whether the discrepancies between perturbative predictions and the high- $q_{T}$ experimental data seen above might be explained by power corrections. We will try here to address this question from a phenomenological point of view.

As a simple way of modeling power corrections we estimate below the impact of a nonperturbative partonic "intrinsic" transverse momentum $k_{T}$ on the Drell-Yan $q_{T}$ spectrum. Such an "intrinsic- $k_{T}$ smearing" is a phenomenological model that has been invoked in the literature in cases where collinear factorization was found to underestimate transverse momentum spectra, like for inclusive prompt photon and pion production in hadronic collisions (see for instance [29, 3, 30]). For inclusive processes such as these and the high- $q_{T}$ Drell-Yan process considered here, no general factorization theorem is known that would extend to arbitrary kinematics of the partonic process. For prompt photons, factorization has been established, however, for near-threshold kinematics and low $k_{T}$ in the framework of the "joint resummation" formalism [31, 32, 33], and for high-energy (small-x) dynamics [34]. A technical challenge for all these approaches is the potential for an artificial singularity when the total transverse momentum of the initial state partons is comparable to the observed transverse momentum. A method for dealing with this issue was proposed in Ref. [35] and found to give rise to power corrections to the cross section. A full treatment of the Drell-Yan cross section may require implementation of perturbative joint resummation along with a study of corrections in inverse powers of $Q$ or $q_{T}$. Rather than pursuing this elaborate framework, for the purpose of obtaining a simple estimate of the potential size of such higher-order perturbative and power-suppressed non-perturbative effects, we resort to an implementation of a simple model of intrinsic- $k_{T}$ smearing. In the following we summarize the main features of the model (the detailed description can be found in Sec. V of [10]). The collinear factorization formula for the process $h_{1} h_{2} \rightarrow \gamma^{*} X$ reads at $\mathrm{LO}\left(\mathscr{O}\left(\alpha_{s}\right)\right)$ :

$$
E \frac{d^{3} \sigma}{d^{3} \mathbf{q}} \equiv \frac{d \sigma}{d y d^{2} \mathbf{q}_{\mathbf{T}}}=\sum_{a, b} \int d x_{a} d x_{b} f_{a / h_{1}}\left(x_{a}, Q^{2}\right) f_{b / h_{2}}\left(x_{b}, Q^{2}\right) \frac{d \hat{\sigma}^{a b \rightarrow \gamma^{*} c}}{d \hat{t}} \frac{\hat{s}}{\pi} \delta\left(\hat{s}+\hat{t}+\hat{u}-Q^{2}\right),
$$

where as before the $f_{a / h}\left(x_{a}, Q^{2}\right)$ are the usual collinear PDFs for partons $a=q, \bar{q}, g$ in hadron $h$. If one allows the incoming partons to have a small transverse momentum $\mathbf{k}_{T}$, Eq. (4.1) becomes [3]:

$$
\begin{aligned}
E \frac{d^{3} \sigma}{d^{3} \mathbf{q}}= & \sum_{a, b} \int d x_{a} d^{2} \mathbf{k}_{a T} d x_{b} d^{2} \mathbf{k}_{b T} F_{a / h_{1}}\left(x_{a}, \mathbf{k}_{a T}, Q^{2}\right) F_{b / h_{2}}\left(x_{b}, \mathbf{k}_{b T}, Q^{2}\right) \\
& \times \frac{\hat{s}}{x_{a} x_{b} s} \frac{d \hat{\sigma}^{a b \rightarrow \gamma^{*} c}}{d \hat{t}} \frac{\hat{s}}{\pi} \delta\left(\hat{s}+\hat{t}+\hat{u}-Q^{2}\right),
\end{aligned}
$$

where the functions $F_{a / h}$ are a generalization of the PDFs, including a dependence on transverse momentum. Notice that the partonic Mandelstam invariants must be modified with the inclusion of $\mathbf{k}_{T}$, and consequently a factor $\hat{s} /\left(x_{a} x_{b} s\right)$ must be inserted to account for the modification of the partonic flux (see Appendix A of [3]). The modification of the partonic four-momenta is most often done according to two criteria: (1) the partons remain on-shell: $p_{a \mu} p_{a}^{\mu}=0$, and (2) the lightcone momentum fractions retain the usual meaning, e.g.: $x_{a}=p_{a}^{+} / P_{a}^{+}$. This leads to the following 
choice, in terms of Minkowski components [36, 3]:

$$
p_{a}^{\mu} \doteqdot\left(x_{a} \frac{\sqrt{s}}{2}+\frac{k_{a T}^{2}}{2 x_{a} \sqrt{s}}, \mathbf{k}_{a T}, x_{a} \frac{\sqrt{s}}{2}-\frac{k_{a T}^{2}}{2 x_{a} \sqrt{s}}\right)
$$

and likewise for the other parton's momentum. Note that we use LO cross sections in Eq. (4.2) since a higher-order formulation is not really warranted for our simple model.

As mentioned above, the framework must become unreliable when $k_{a T}$ or $k_{b T}$ become of the order of the observed transverse momentum, and arguably well before. Large values of $k_{a T}$ can make the partonic Mandelstam in the denominators of the LO hard-scattering cross sections unphysically small. In [3], the following condition was chosen to limit the size of, for example, $k_{a T}$ :

$$
k_{a T}<\min \left[x_{a} \sqrt{s}, \sqrt{x_{a}\left(1-x_{a}\right) s}\right] .
$$

This ensures that each parton moves predominantly along the direction of its parent hadron, and that its energy does not exceed the hadron's energy. However, for $\sqrt{s} \simeq 40 \mathrm{GeV}$ (E866 and E605 experiments), this condition implies that $k_{a T}$ may still reach values as high as $20 \mathrm{GeV}$. In our numerical analysis we therefore prefer to introduce an additional cutoff $k_{T \max }$ on both $k_{a T}$ and $k_{b T}$ and will test the dependence of the results on this cutoff.
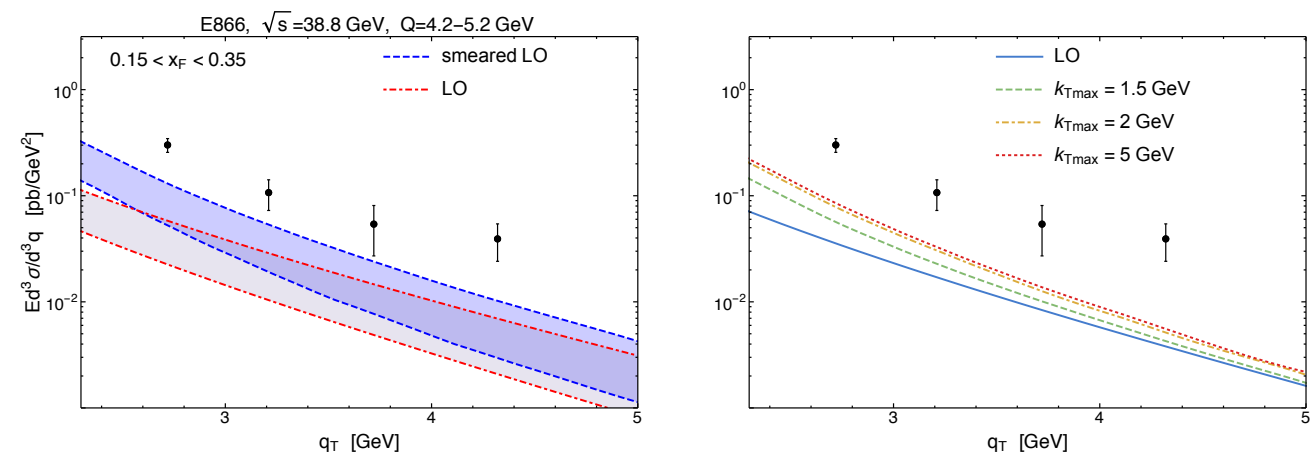

Figure 5: Left panel: the effect of $k_{T}$-smearing (dashed blue lines), with the cutoff $k_{T \max }$ in Eq. (4.2) set to $2 \mathrm{GeV}$. The bands correspond to variation of factorization and renormalization scales between $Q / 2$ and $2 Q$. For comparison, the calculation in ordinary collinear factorization at LO is also shown (red dotted lines). Right panel: the effect of varying the cutoff $k_{T \max }$ in Eq. (4.2). Here the curves correspond to the central values $\mu_{R}=\mu_{F}=Q$. For $k_{T \max } \geq 2 \mathrm{GeV}$, which corresponds to the $99 \%$ percentile of the gaussian in Eq. (4.5), independence from the cutoff is reached.

In Fig. 5 we show the effect of $k_{T}$-smearing, Eq. (4.2), for E866 kinematics. For the generalized PDFs in Eq. (4.2), we use

$$
F_{a / h}\left(x_{a}, \mathbf{k}_{a T}, Q^{2}\right)=f_{a / h}\left(x_{a}, Q^{2}\right) \frac{1}{\pi\left\langle k_{T}^{2}\right\rangle} \exp \left[-\frac{k_{a T}^{2}}{\left\langle k_{T}^{2}\right\rangle}\right],
$$


where $\left\langle k_{T}^{2}\right\rangle$ is independent of flavor ${ }^{2}$ and momentum fraction $x_{a}$, but does depend logarithmically on $Q^{2}$ because of soft gluon radiation. The $\left\langle k_{T}^{2}\right\rangle$ of the Gaussian is chosen by comparing to the TMD fit in [7] (see discussion below Fig. 14 in [10]). The impact of smearing on the cross section overall remains mild, as long as the cutoff $k_{T \max }$ is chosen below $2 \mathrm{GeV}$. Especially the regime $q_{T} \simeq Q$ is only little affected by $k_{T}$-smearing. We conclude that, although $k_{T}$-smearing does somewhat improve the comparison with the data, its effects do not appear to be sufficiently large to lead to a satisfactory agreement. We note that at lower c.m.s. energies as relevant for E288, one is forced to choose smaller cutoffs since the reach in $q_{T}$ is more limited in these cases.

\section{Conclusions}

We have shown that theoretical predictions based on fixed-order perturbation theory fail to describe Drell-Yan data at low c.m. energy $\sqrt{s} \sim 40 \mathrm{GeV}$ and large values $q_{T} \sim Q$ of the transverse momentum of the lepton pair, the experimental cross sections being significantly larger than the theoretical ones. This is the region where collinear-factorized perturbation theory is expected to accurately describe the cross section. This disagreement is observed for different experiments, and across a range of different kinematics in $x_{F}, y$ and $Q$ (see Sec. III of [10]), although admittedly the experimental uncertainties are in some cases quite large.

Barring the possibility of sizable normalization uncertainties in the experiments, it is important to identify the theoretical origins of the discrepancies observed in the fixed-target regime. We have first implemented perturbative threshold resummation and found that it improves the situation somewhat; a significant discrepancy remains, however. This leaves the investigation of powersuppressed corrections, which we have modeled by implementing a simple Gaussian intrinsic- $k_{T}$ smearing into the LO cross section. We find that this again helps somewhat, but does not lead to a satisfactory description of the data. Ultimately, a more detailed study of power corrections may be required in this case.

Our findings are in line with those reported for the SIDIS cross section in Ref. [9]. We close by stressing the importance of obtaining a thorough understanding of the full Drell-Yan and SIDIS $q_{T}$-spectra in the fixed-target regime. Low- $q_{T}$ Drell-Yan and SIDIS cross sections measured at fixed-target experiments are a prime source of information on TMDs. At present, the theoretical description for the important "matching regime" around $q_{T}=2 \mathrm{GeV}$ is not robust (see Sec. II in [10]). Given the shape of the experimental spectra, it appears that TMD physics may extend to such large $q_{T}$ and may well remain an important ingredient even beyond. This view is corroborated by the fact that the $q_{T}$-integrated Drell-Yan cross section is well described by fixed-order perturbation theory at these energies. In any case, a reliable interpretation of data in terms of TMDs, including the matching to collinear physics, is only possible if the cross sections are theoretically understood over the full transverse-momentum range, which includes the regime of $q_{T} \sim Q$ we have addressed here.

\footnotetext{
${ }^{2}$ We remark that the initial parton " $a$ " can also be a gluon. Every $k_{T}$-smearing model has to make an assumption for the average gluon transverse momentum, which is usually taken to be the same as that for the quarks. We note that perturbative resummations predict dependence of $\left\langle k_{T}^{2}\right\rangle$ on parton flavor [35].
} 


\section{Acknowledgments}

AB, GB and FP acknowledge support from the European Research Council (ERC) under the European Union's Horizon 2020 research and innovation program (grant agreement No. 647981, 3DSPIN). This work has been supported in part by the Bundesministerium für Bildung und Forschung (BMBF) under grant no. 05P15VTCA1.

\section{References}

[1] F. Landry, R. Brock, G. Ladinsky and C. P. Yuan, New fits for the nonperturbative parameters in the CSS resummation formalism, Phys. Rev. D63 (2001) 013004 [hep-ph/9905391].

[2] F. Landry, R. Brock, P. M. Nadolsky and C. P. Yuan, Tevatron Run-1 Z boson data and Collins-Soper-Sterman resummation formalism, Phys. Rev. D67 (2003) 073016 [hep-ph/0212159].

[3] U. D'Alesio and F. Murgia, Parton intrinsic motion in inclusive particle production: Unpolarized cross sections, single spin asymmetries and the Sivers effect, Phys. Rev. D70 (2004) 074009 [hep-ph/0408092].

[4] A. V. Konychev and P. M. Nadolsky, Universality of the Collins-Soper-Sterman nonperturbative function in gauge boson production, Phys. Lett. B633 (2006) 710 [hep-ph/ 0506225$].$

[5] U. D'Alesio, M. G. Echevarria, S. Melis and I. Scimemi, Non-perturbative QCD effects in $q_{T}$ spectra of Drell-Yan and Z-boson production, JHEP 11 (2014) 098 [1407.3311].

[6] P. Sun, J. Isaacson, C. P. Yuan and F. Yuan, Nonperturbative functions for SIDIS and Drell-Yan processes, Int. J. Mod. Phys. A33 (2018) 1841006 [1406.3073].

[7] A. Bacchetta, F. Delcarro, C. Pisano, M. Radici and A. Signori, Extraction of partonic transverse momentum distributions from semi-inclusive deep-inelastic scattering, Drell-Yan and Z-boson production, JHEP 06 (2017) 081 [1703.10157].

[8] I. Scimemi and A. Vladimirov, Analysis of vector boson production within TMD factorization, Eur. Phys. J. C78 (2018) 89 [1706.01473].

[9] J. O. Gonzalez-Hernandez, T. C. Rogers, N. Sato and B. Wang, Challenges with Large Transverse Momentum in Semi-Inclusive Deeply Inelastic Scattering, Phys. Rev. D98 (2018) 114005 [1808.04396].

[10] A. Bacchetta, G. Bozzi, M. Lambertsen, F. Piacenza, J. Steiglechner and W. Vogelsang, Difficulties in the description of Drell-Yan processes at moderate invariant mass and high transverse momentum, 1901.06916.

[11] W. Bizon, X. Chen, A. Gehrmann-De Ridder, T. Gehrmann, N. Glover, A. Huss et al., Fiducial distributions in Higgs and Drell-Yan production at $N^{3} L L+N N L O, 1805.05916$.

[12] A. S. Ito et al., Measurement of the Continuum of Dimuons Produced in High-Energy Proton Nucleus Collisions, Phys. Rev. D23 (1981) 604.

[13] NuSEA collaboration, Measurement of the light anti-quark flavor asymmetry in the nucleon sea, Phys. Rev. Lett. 80 (1998) 3715 [hep-ex/9803011].

[14] J. C. Webb, Measurement of continuum dimuon production in 800-GeV/C proton nucleon collisions, Ph.D. thesis, New Mexico State U., 2003. hep-ex/ $0301031.10 .2172 / 1155678$. 
[15] A. D. Martin, W. J. Stirling, R. S. Thorne and G. Watt, Parton distributions for the LHC, Eur. Phys. J. C63 (2009) 189 [0 901.0002 ].

[16] NNPDF collaboration, Parton distributions from high-precision collider data, Eur. Phys. J. C77 (2017) 663 [1706.00428].

[17] P. B. Arnold and R. P. Kauffman, $W$ and $Z$ production at next-to-leading order: From large $q T$ to small, Nuclear Physics B 349 (1991) 381 .

[18] S. Dulat, T.-J. Hou, J. Gao, M. Guzzi, J. Huston, P. Nadolsky et al., New parton distribution functions from a global analysis of quantum chromodynamics, Phys. Rev. D93 (2016) 033006 [1506.07443].

[19] G. F. Sterman, Summation of Large Corrections to Short Distance Hadronic Cross-Sections, Nucl. Phys. B281 (1987) 310.

[20] S. Catani and L. Trentadue, Comment on QCD exponentiation at large x, Nucl. Phys. B353 (1991) 183.

[21] D. de Florian and W. Vogelsang, Threshold resummation for the inclusive-hadron cross-section in pp collisions, Phys. Rev. D71 (2005) 114004 [hep-ph/ 0501258$].$

[22] P. Hinderer, F. Ringer, G. Sterman and W. Vogelsang, Threshold Resummation at NNLL for Single-particle Production in Hadronic Collisions, 1812.00915.

[23] N. Kidonakis and V. Del Duca, Electroweak boson hadroproduction at large transverse momentum: Factorization, resummation, and NNLO corrections, Phys. Lett. B480 (2000) 87 [hep-ph/9911460].

[24] N. Kidonakis and A. Sabio Vera, W hadroproduction at large transverse momentum beyond next-to-leading order, JHEP 02 (2004) 027 [hep-ph/ 0311266 ].

[25] R. J. Gonsalves, N. Kidonakis and A. Sabio Vera, $W$ production at large transverse momentum at the large hadron collider, Phys. Rev. Lett. 95 (2005) 222001 [hep-ph / 0507317$].$

[26] N. Kidonakis and R. J. Gonsalves, NNLO soft-gluon corrections for the Z-boson and W-boson transverse momentum distributions, Phys. Rev. D89 (2014) 094022 [1 404.4302 ].

[27] C. Muselli, S. Forte and G. Ridolfi, Combined threshold and transverse momentum resummation for inclusive observables, JHEP 03 (2017) 106 [1701.01464].

[28] D. de Florian, A. Kulesza and W. Vogelsang, Threshold resummation for high-transverse-momentum Higgs production at the LHC, JHEP 02 (2006) 047 [hep-ph / 0511205].

[29] J. Huston, E. Kovacs, S. Kuhlmann, H. L. Lai, J. F. Owens and W. K. Tung, A Global QCD study of direct photon production, Phys. Rev. D51 (1995) 6139 [hep-ph/9501230].

[30] L. Apanasevich et al., $k_{T}$ effects in direct photon production, Phys. Rev. D59 (1999) 074007 [hep-ph/9808467].

[31] E. Laenen, G. F. Sterman and W. Vogelsang, Recoil and threshold corrections in short distance cross-sections, Phys. Rev. D63 (2001) 114018 [hep-ph/ 0010080$].$

[32] E. Laenen, G. F. Sterman and W. Vogelsang, Higher order QCD corrections in prompt photon production, Phys. Rev. Lett. 84 (2000) 4296 [hep-ph / 0002078 ].

[33] H.-n. Li, Unification of the $k(T)$ and threshold resummations, Phys. Lett. B454 (1999) 328 [hep-ph/9812363]. 
[34] M. A. Kimber, A. D. Martin and M. G. Ryskin, Unintegrated parton distributions and prompt photon hadroproduction, Eur. Phys. J. C12 (2000) 655 [hep-ph/9911379].

[35] G. F. Sterman and W. Vogelsang, Recoil and power corrections in high-x(T) direct-photon production, Phys. Rev. D71 (2005) 014013 [hep-ph/ 0409234 ].

[36] J. F. Owens, Large-momentum-transfer production of direct photons, jets, and particles, Rev. Mod. Phys. 59 (1987) 465. 